\title{
A new perspective on Bruch's membrane and the retinal pigment epithelium*
}

\author{
MICHAEL H. GOLDBAUM AND KENDALL MADDEN
}

From the Division of Ophthalmology at Veterans Administration Medical Center and University of California, San Diego, California, USA

SUMMARY Trypsin digestion of retinal pigment epithelium is a technique that bares Bruch's membrane, to allow topographical examination by scanning electron microscopy. Twenty-five human eyes were examined. The zonula occludens of the pigment epithelium was clearly seen as a surface feature, but attachment plaques at the sides and base were not visible. The adhesion between the pigment epithelium and the basal lamina was stronger than between the basal lamina and the rest of Bruch's membrane. Surface features of the basal lamina, inner collagenous zone, elastic layer, and outer collagenous zone were seen in a way that previously required an artist's representation constructed from microscopic sections.

Since 1844, when Karl Bruch described a 'structureless membrane' between the retina and choroid, ${ }^{1}$ almost all anatomical examinations have been by light microscopy or transmission electron microscopy. ${ }^{2 \rightarrow}$ Most of these histological and electron microscopic sections were cross-sections or tangential sections through the membrane, which we describe today as having 5 components: (1) basal lamina of the pigment epithelium; (2) inner collagenous layer; (3) elastic layer; (4) outer collagenous layer; and (5) basal lamina of the choriocapillaris, where it is present.

A technique was devised to loosen enzymatically pigment epithelium from Bruch's membrane to allow evaluation of surface features by scanning electron microscopy, thereby offering a perspective view of Bruch's membrane and its component layers. This technique also demonstrates surface features of pigment epithelium that have not been demonstrated previously. Scanning electron microscopy allows conceptualisation of 3-dimensional structure in a way that sections cannot. In this report the surface features of Bruch's membrane and parts of the retinal pigment are described. In separate reports the same techniques will be used to evaluate regional variations, drusen, and aging changes in Bruch's membrane.

Correspondence to Michael H. Goldbaum, MD, 225 Dickinson Street (H-898) San Diego, California 92103, USA.

*Presented in part at the Association for Research in Vision and Ophthalmology. Sarasota, Florida, 4 May 1979.

\section{Methods and materials}

Twenty-five eyes were obtained from the eye bank and placed in chilled buffered glutaraldehyde $1 \%$ and paraformaldehyde $1 \%$ (buffered to $\mathrm{pH} 7.45$ with $0 \cdot 1 \mathrm{M}$ monobasic and dibasic phosphate) between 50 minutes and 3 hours 25 minutes after death. The corneas of most of the eyes were removed prior to fixation. The donors ranged in age from 15 to 83 years.

After fixation the eyes were sectioned coronally through the equator. Each calotte was examined with the stereoscopic microscope for drusen, pigment changes, and other abnormalities. Three specimens were obtained with trephines. A specimen containing the disc and macular region was obtained with an $8 \cdot 5$ $\mathrm{mm}$ trephine. A specimen at the equator and a specimen straddling the ora each were obtained with a $6 \mathrm{~mm}$ trephine.

To help localisation of the region under the fovea the piece containing disc and macula was notched at the edge superior and inferior to the fovea. The sensory retina was removed, and each piece was photographed.

After the retina was removed each trephined piece was digested in buffered bovine pancreas trypsin $0.5 \%$ (Sigma) for 40 to 60 minutes at $37^{\circ} \mathrm{C}$. The trypsin was buffered to pH $7 \cdot 25$ with $0 \cdot 1 \mathrm{M}$ Tris maleate buffer in $0.02 \%$ ethylene diamine tetra-acetic acid. The retinal pigment epithelium was washed off Bruch's membrane with a stream of phosphate 
Fig. 1 Scanning electron microscopy of pigment epithelium showing smooth side walls and microvilli on inner surface.

Pockets in microvilli surrounded rods. Microvilli at border of pockets (arrow) mat together to form ridges that surround tip of rod. $\mathrm{D}=$ druse. $(\times 1745)$.

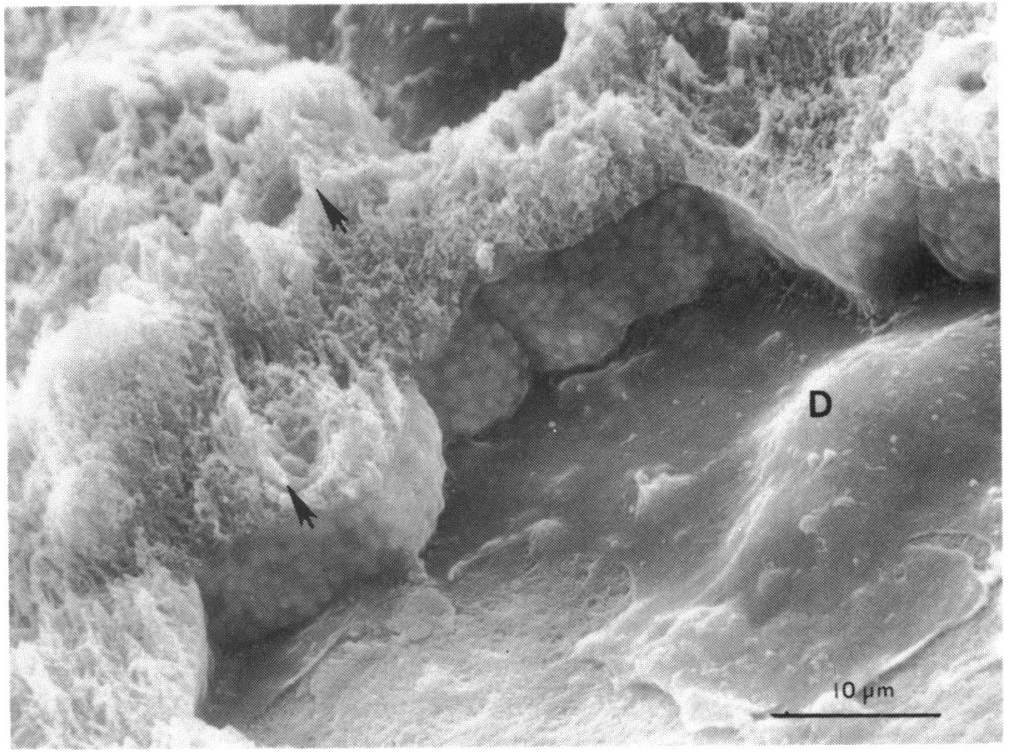

microscopy of the same piece. After a specimen was examined by scanning microscopy it was placed in ethanol $100 \%$, then mounted in Epon 812 for thin sectioning and staining. ${ }^{10}$ Other pieces were cut in half for separate processing for scanning electron microscopy and transmission electron microscopy.

\section{Results}

\section{PIGMENT EPITHELIUM}

The digestion process loosened most of the pigment epithelium from Bruch's membrane; irrigation with and uranyl acetate. Some specimens were examine by correlated scanning and transmission electron

Fig. 2 Transmission electron microscopy of pigment epithelium and Bruch's membrane.

$\mathrm{MV}=$ microvilli, $\mathrm{RPE}=$ pigment epithelium, $\mathrm{BI}=$ basal infolding, $\mathrm{BLR}=$ basal lamina of pigment epithelium, $\mathrm{ICZ}=$ inner collagenous zone, $\mathrm{EL}=$ elastic lamina, $\mathrm{OCZ}=$ outer collagenous zone, BLE = basal lamina of endothelium of choriocapillaris. $(\times 8290)$.

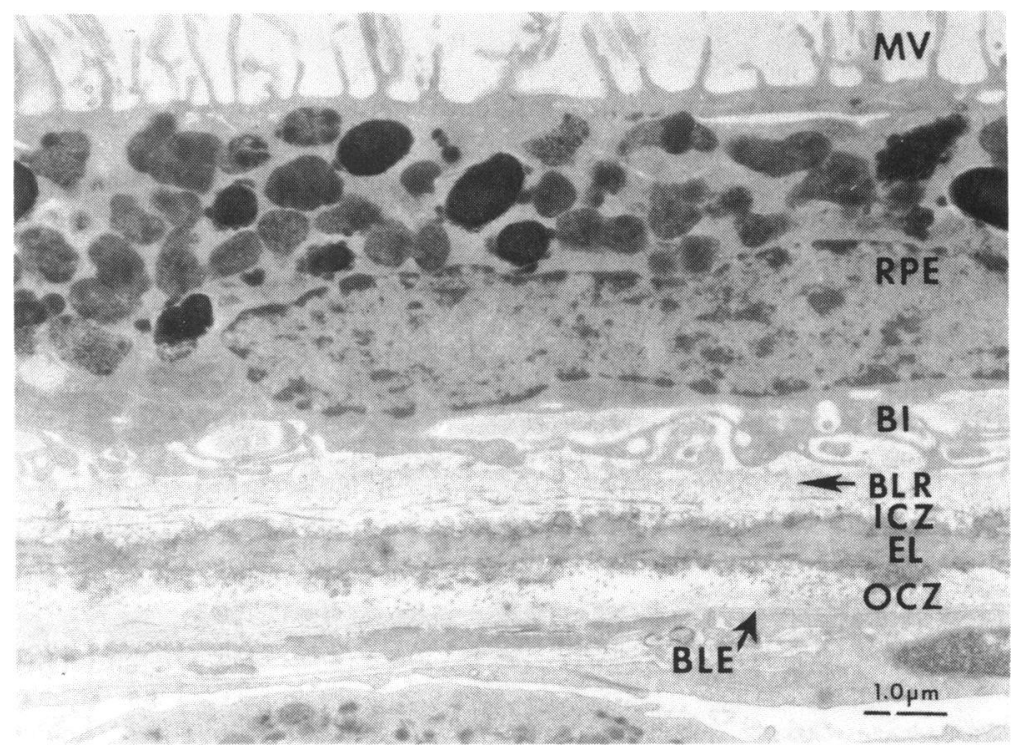




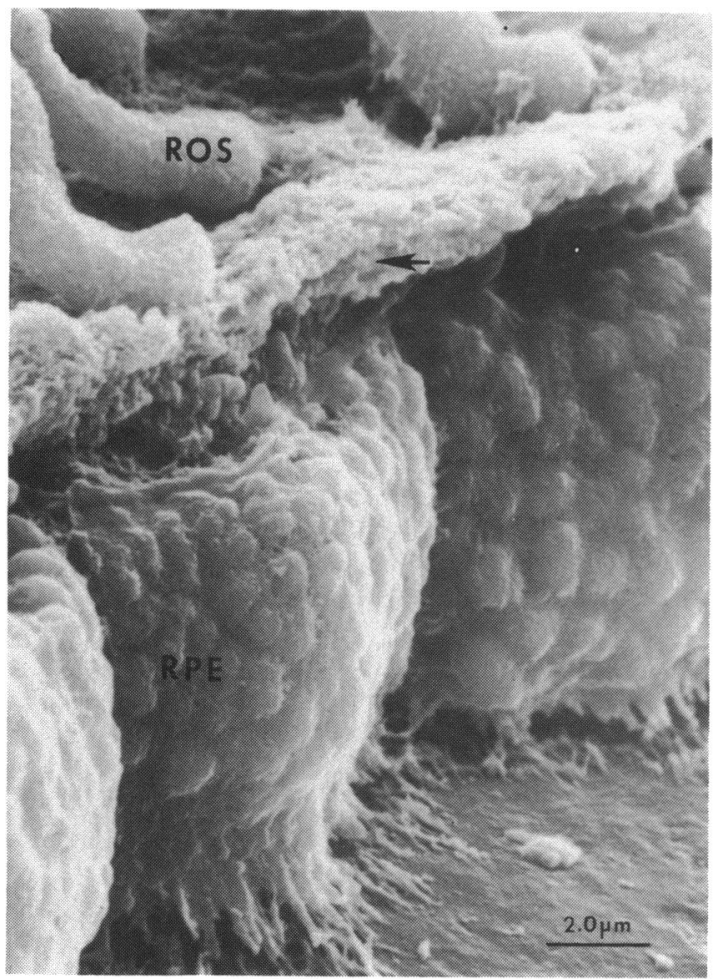

Fig. 3 Rods inserting into plate of material on apical surface of retinal pigment epithelium (arrow). ROS $=$ rod outer segment. (Magnification $=\times 5965)$. phosphate buffer exposed broad expanses of Bruch's membrane. Patches of pigment epithelium remained attached to Bruch's membrane.

Microvillous processes extended from the apex of the pigment epithelial cells (Figs. 1 and 2). In most of the specimens the retina completely separated from the pigment epithelium, leaving pockets where the outer receptors of the rods had been. The microvilli were long and hair-like between these pockets. ${ }^{11}$ At the border of the pockets the villous processes appeared matted together or took the form of a ridge partially wrapped around the pocket. In some specimens the villous processes were covered with a material, presumably the mucopolysaccharides between outer receptors and the pigment epithelium. This material appeared as a plate with spaces for the receptors (Fig. 3).

The site of the zonula occludens was visible as a band about $500 \mathrm{~nm}$ wide encircling each pigment cell at the junction between the apex and the sides (Fig. 4). In most specimens this band was a flat outpouching of the cell membrane at the sides of the pigment cell. In other specimens this band appeared to shrink from processing more than the sides of the cell, which sometimes bulged above the band.

The zonula adherens and the maculae adherentes were not visible on the sides of the pigment epithelium by scanning electron microscopy. The smooth cell membrane on the sides of the pigment epithelium bulged over the melanin granules (Fig. 1).

The basal surface of the pigment epithelium was generally not visible from the outside. In one specimen a sheet of remaining pigment epithelium was detached from the basal lamina and curled

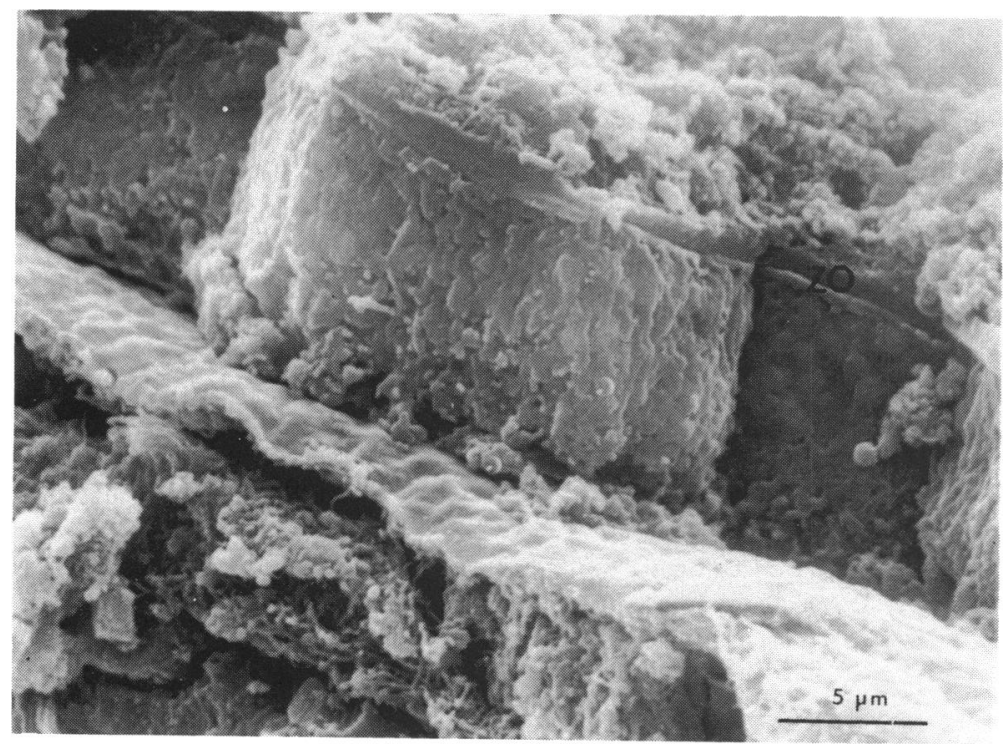

Fig. 4 Pigment epithelium with a band at site of zonula occludens (ZO) surrounding each cell at junction between sides and apex. The sides show some deterioration from exposure to trypsin. $(\times 3117)$. 


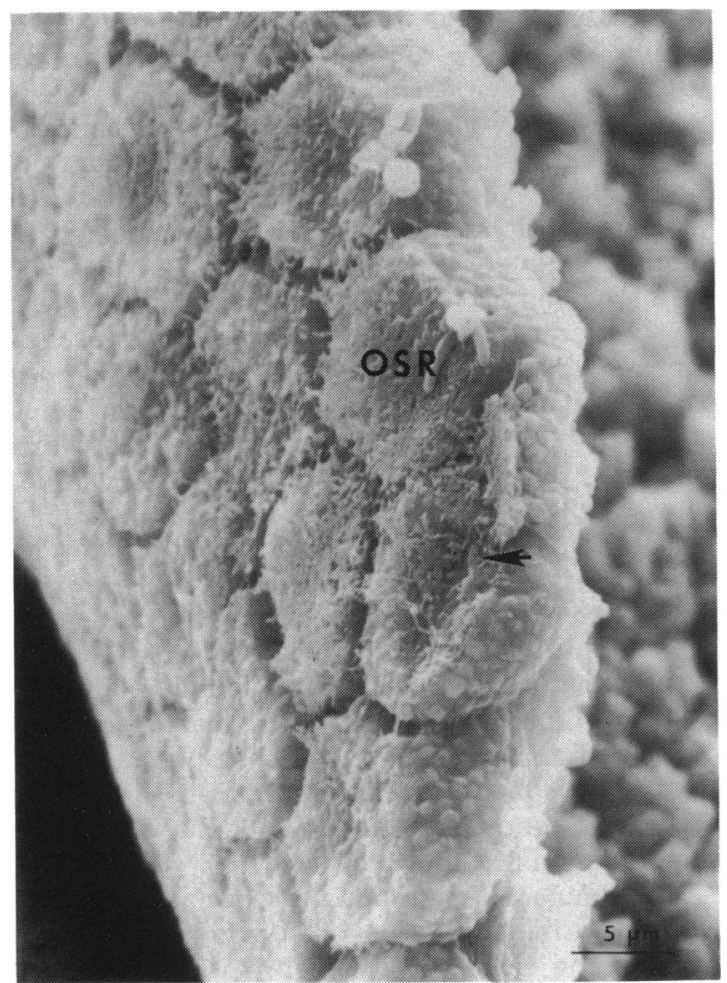

Fig. 5 Sheet of pigment epithelium that has peeled up from basal lamina. An amorphous material coats outer surface of pigment epithelium (OSR). Some basal infolding is visible (arrow). $(\times 2147)$.

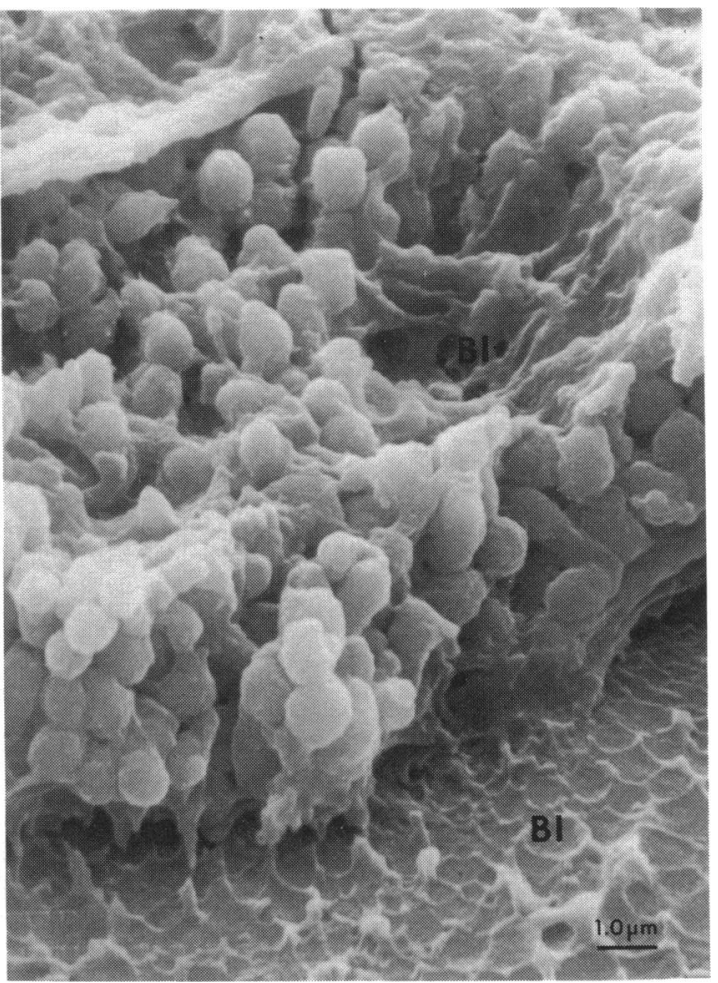

Fig. 6 Partially digested pigment epithelium. Remnant basal infoldings (BI) on the basal lamina show that adhesion between basal lamina and basal infolding was stronger than integrity of cell in this eye. $(\times 5965)$.
Fig. 7 Remnants of basal infoldings attached by attachment plaques (arrow) to pigment epithelium. $(\times 10286)$.

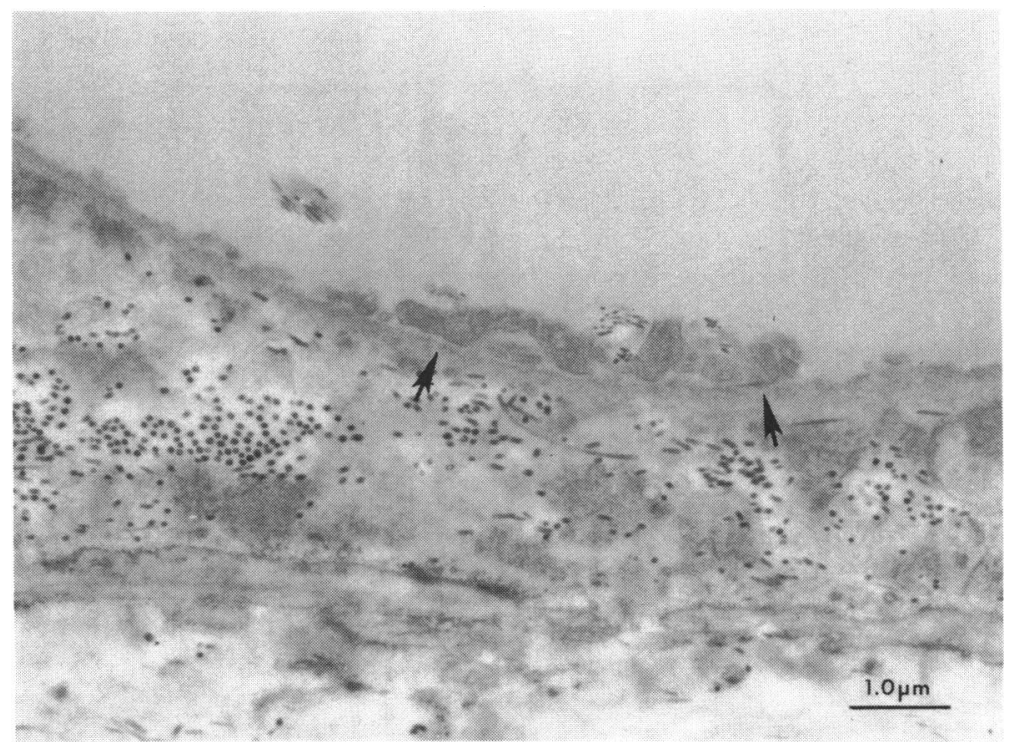




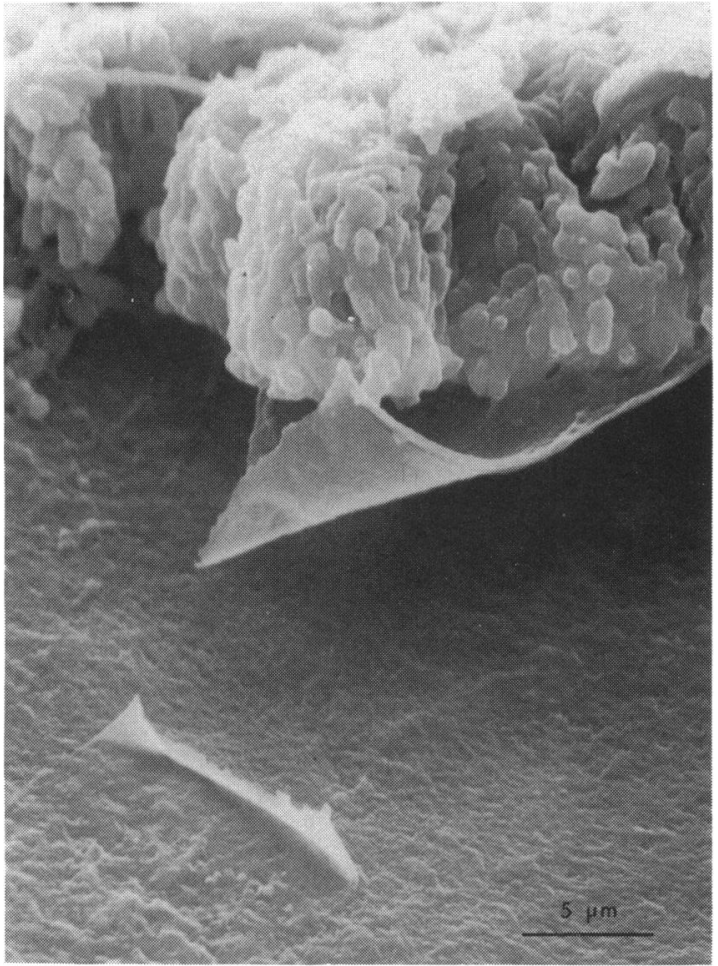

Fig. 8 Basal lamina peeling away from inner collagenous zone. At low power basal lamina appears like a plastic membrane. $(\times 2684)$.

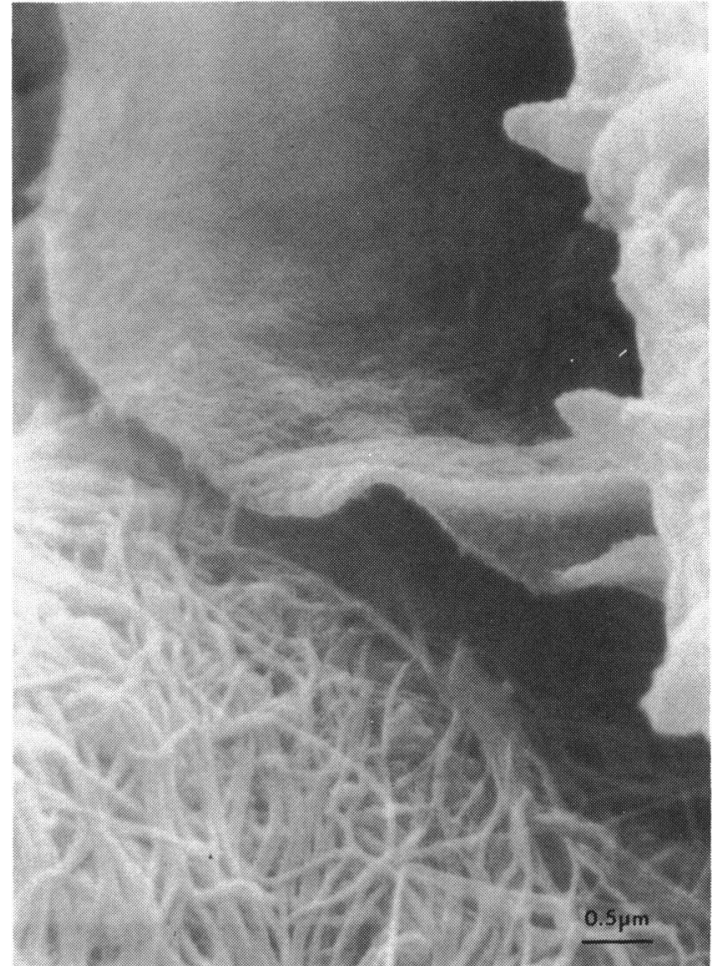

Fig. 9 Basal lamina at high magnification appears granular or velvety. $(\times 14316)$. 


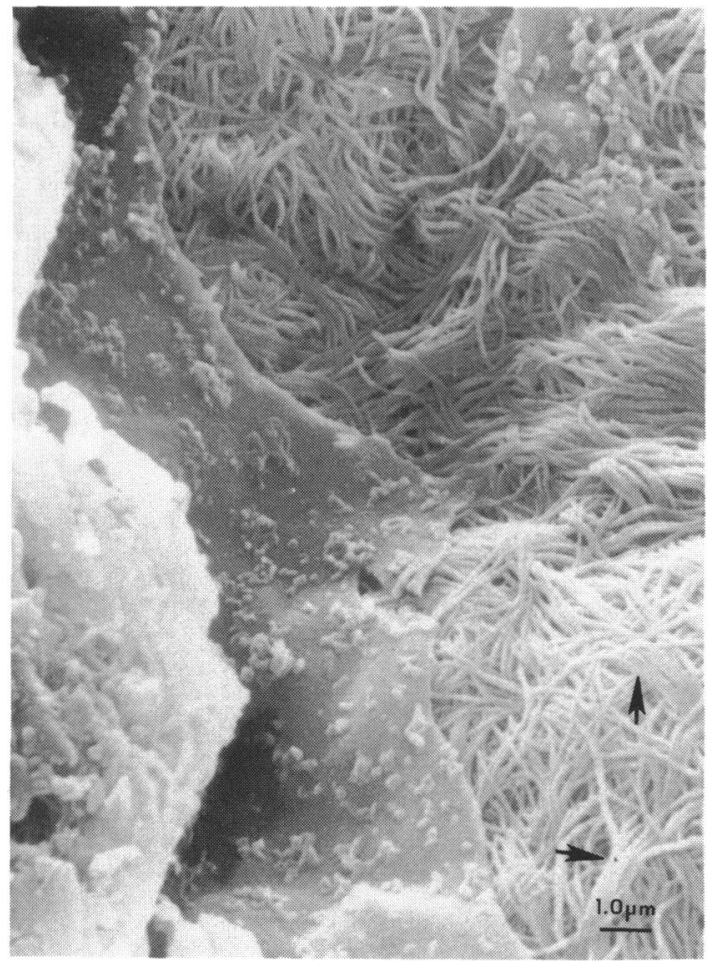

Fig. 11 Where basal lamina is absent, the inner collagenous zone is visible, revealing tight interweaving of collagen fibres. The fibres occasionally appear joined together lengthwise (arrow). $(\times 5368)$.

inwards. An amorphous material adhered to the external surface, leaving clefts where basal infolding was present (Fig. 5).

\section{PIGMENT EPITHELIUM ADHESIONS TO BRUCH'S MEMBRANE}

In many specimens the adhesion of the basal cell membrane to the basal lamina of Bruch's membrane was stronger than the integrity of the cell, leaving remnant basal cell membrane on the basal lamina. The internal aspect of this remnant membrane showed basal infolding (Fig. 6). Transmission electron' microscopy of the same piece showed attachment plaques of the flat parts of the cell basal membrane to the basal lamina (Fig. 7).

Broad expanses of the basal lamina were completely bared following enzymatic digestion and irrigation. The inner surface of the basal lamina was smooth and showed no evidence of the location of attachment plaques of pigment epithelium (Figs. 8 and 9).

BRUCH'S MEMBRANE

Four of the 5 layers of Bruch's membrane were

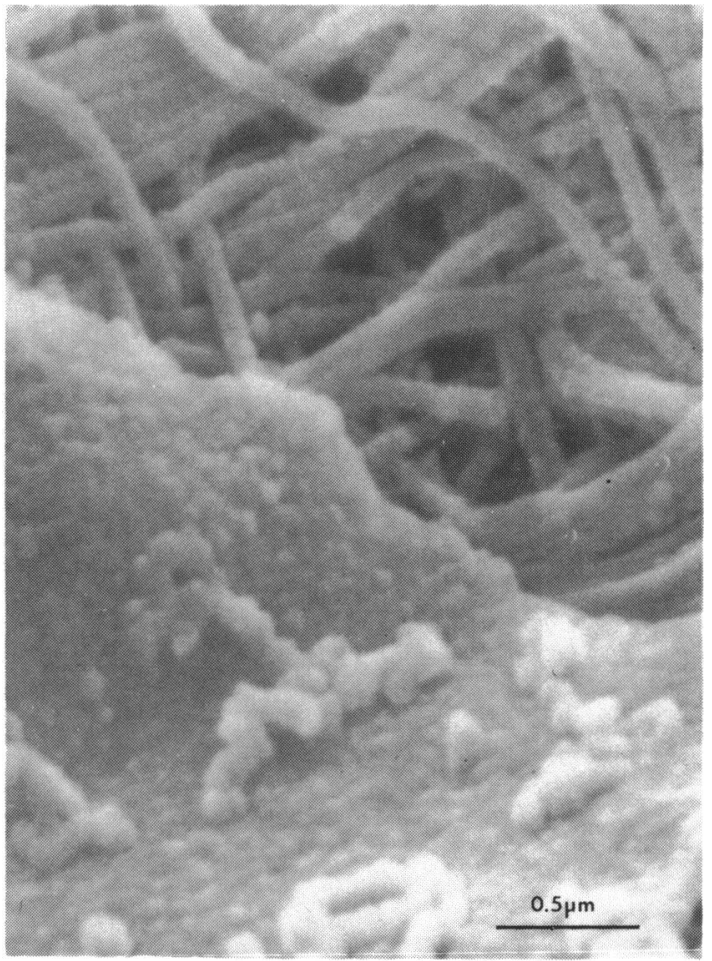

Fig. 12 With high magnification the fibres of the inner collagenous zone appear to have an external representation of banding. $(\times 29825)$.

observed by scanning electron microscopy. The process of enzymatic digestion and irrigation exposed most of Bruch's membrane. 25 to $50 \%$ of the basal lamina was lost from each specimen, allowing easy visualisation of the inner collagenous zone. The rest of Bruch's membrane generally remained intact. The shrinkage produced by the critical-point processing caused the edges of some specimens of Bruch's membrane to curl inwards, allowing visualisation of the elastic layer and the outer collagenous layer. The basal lamina of the choriocapillaris endothelium was not seen.

Basal lamina of the pigment epithelium. By transmission electron microscopy the basal lamina showed as a straight, finely-granular membrane 40 to $100 \mathrm{~nm}$ thick (Fig. 1). Its appearance by transmission electron microscopy did not prepare one for its appearance by scanning electron microscopy. With moderate magnification the basal lamina appeared like a smooth thin plastic membrane (Fig. 8). At high magnification, the membrane took on a more granular appearance (Fig. 9). Both surfaces were smooth. There was no visible evidence on the inner surface where pigment epithelial attachments were located. There was no evidence of 


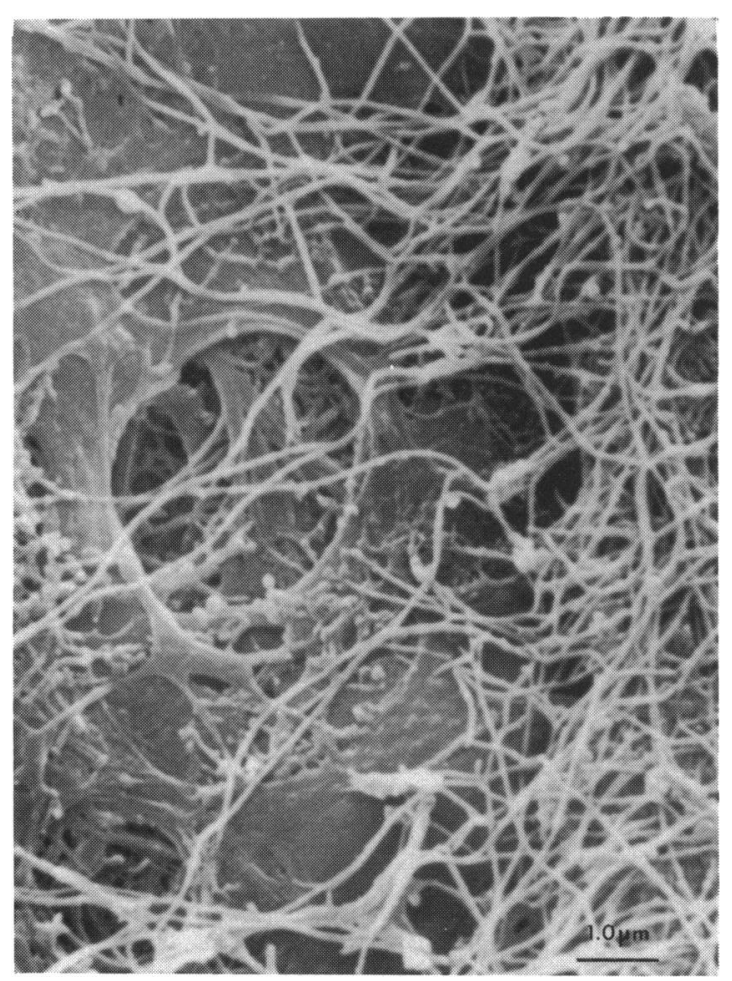

Fig. 13 Elastic lamina with some collagen fibres lying on inner surface. Some of inner collagen fibres are inserted into elastic layer and some join outer collagenous layer by passing through holes in elastic lamina. $(\times 8350)$.

insertion of collagen fibrils from the inner collagenous layer into the basal lamina (Fig. 10). The basal lamina tended to separate easily from the inner collagenous layer; the attachment between the basal lamina and the inner collagenous layer appeared to be much less firm than between the pigment epithelium and the basal lamina (Figs. 6, 10).

Inner collagenous layer. At low magnification the inner collagenous layer appeared like velvet (Fig. 8). At moderate magnification the collagen fibres in the inner collagenous layer formed a tightly interwoven membrane (Fig. 11). The ground substance between the fibres was not visible. At high magnification the collagen fibres appeared to have a uniform thickness of about $70 \mathrm{~nm}$ (Fig. 12). There were transverse ridges with a periodicity of about $60 \mathrm{~nm}$, perhaps representing a surface manifestation of the banding seen in these fibres by transmission electron microscopy.

Elastic layer. The elastic layer appeared to be composed of coarse and fine fibres matted together by some amorphous substance (Fig. 13). This layer formed an almost solid sheet with clefts and small holes. Collagen fibres from the inner collagenous

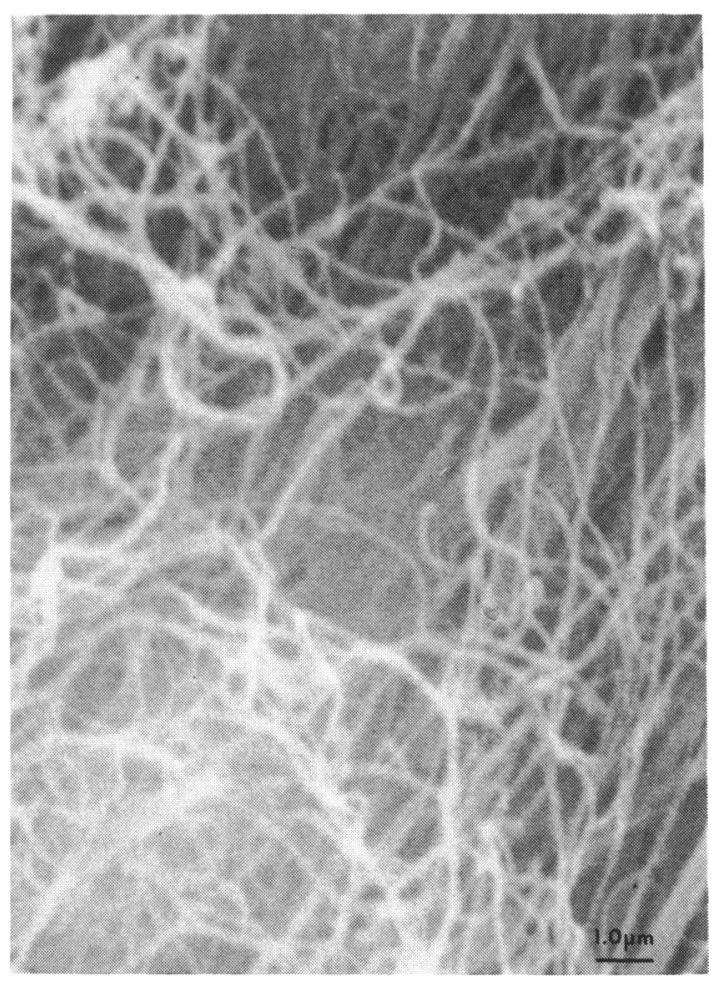

Fig. 14 Curling up of part of Bruch's membrane has exposed outer collagenous layer. The collagen fibres are less tightly woven. $(\times 5276)$.

layer appeared to be inserted into the elastic layer and also to pass through holes in the elastic lamina to join with collagen fibres in the outer collagenous layers.

Outer collagenous layer. The collagen fibres in the outer collagenous layer were less dense, finer in appearance, and more loosely interwoven than those in the inner collagenous layer (Fig. 14). The collagen network between the choriocapillaries appeared similar to the outer collagenous layer, with which it was continuous (Fig. 15).

\section{Discussion}

The enzymatic loosening of the pigment epithelium allowed scanning electron microscopy of Bruch's membrane. Some of the pigment epithelium did not wash away, and the remaining cells showed variable amounts of enzyme effect, ranging from not visible to severely digested.

The microvilli around receptor elements have previously been observed by transmission electron microscopy to mat together and form ramparts. ${ }^{9}$ The ridge we saw with scanning electron microscopy may 
Fig. 15 Outer collagenous layer is continuous with collagen fibres between vessels in choroid. Split in Bruch's membrane has exposed choriocapillaris and collagen fibres between. $(\times 3517)$.

be the same feature. On the other hand there could be an enzyme artefact. Likewise the plate we sometimes observed between pigment epithelium and rods and cones could be mucopolysaccharides or could be an enzyme artefact. The zonula occludens was a surface feature visible as a band round the sides of the cell near the apex. ${ }^{12}{ }^{13} \mathrm{We}$ believe the zonula occludens has not previously been described as a visible surface feature of the retinal pigment epithelium. It functions as an outer blood-retina barrier to large molecules. ${ }^{14-16}$ The sides of the pigment epithelium covered the pigment granules and were otherwise smooth; attachment plaques at the sides

Fig. 16 Artist's representation of pigment epithelium and Bruch's membrane. Microvilli extend from apical surface of pigment epithelium, occasionally forming pockets for rod outer segment. The zonula occludens barrier surrounds each cell, separating the side membrane and the apical surface. The basal lamina of the pigment epithelium is smooth like a thin plastic membrane. Basal infoldings adhere to basal lamina, which is not tightly attached to the inner collagenous zone. Inner collagenous fibres are more tightly woven than outer collagenous fibres. Elastic lamina forms a plate with holes between the 2 collagenous zones.
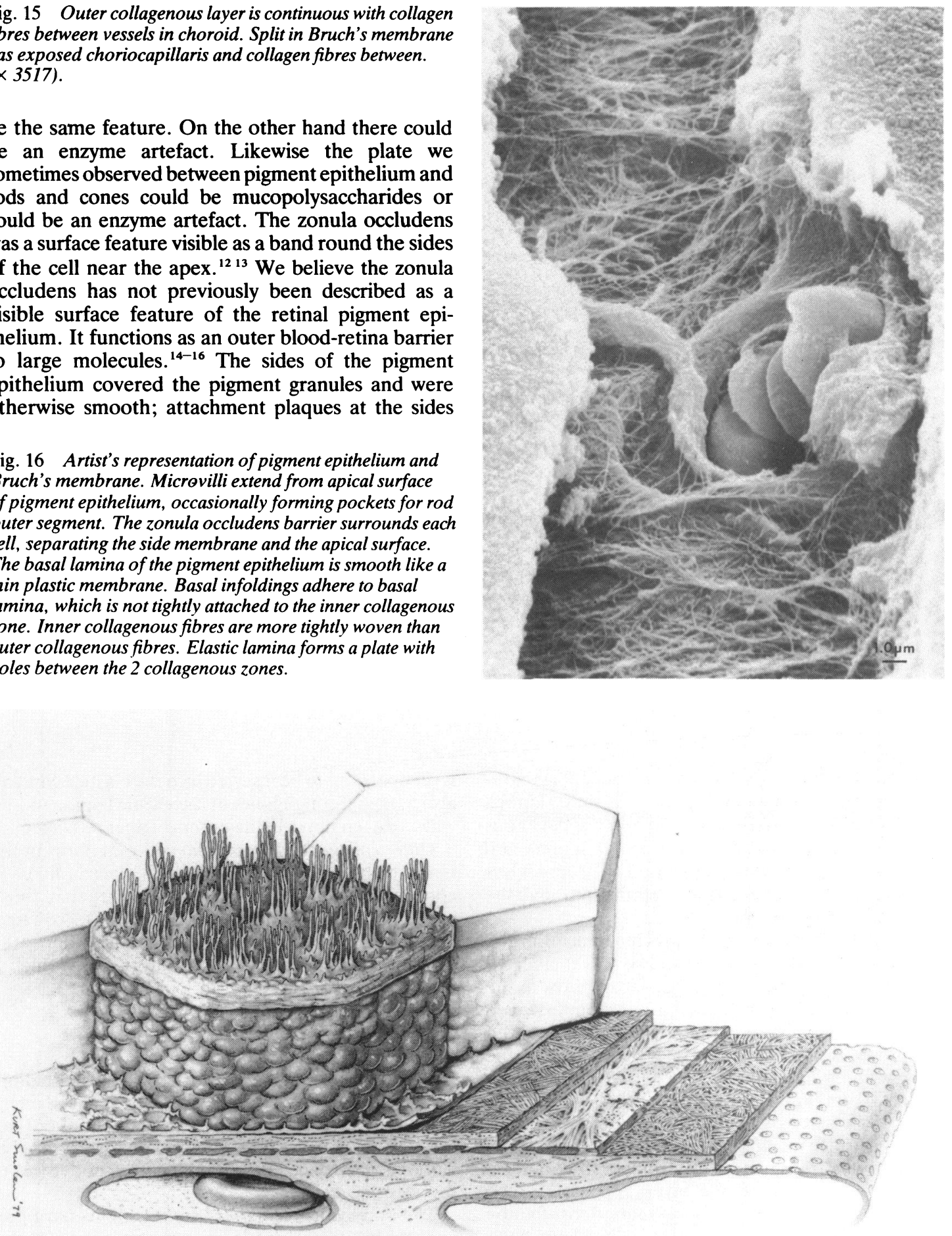
of the pigment epithelium ${ }^{17}$ did not produce visible changes on the surface.

The adhesion between retinal pigment epithelium and the basal lamina was stronger than between the basal lamina and the collagenous layer. This latter junction is also the site of separation for the clinical condition of retinal pigment epithelial detachment. Attachment plaques at the basal layer of the pigment epithelium were seen in transmission electron microscopy, but were not visible as surface features by scanning electron microscopy. The amorphous material observed on the outer surface of the cell basal membrane (Fig. 10) may be involved in the adhesion to the basal lamina. There was no visible mechancal attachment between the smooth outer surface of the basal lamina and the rest of Bruch's membrane (Fig. 10).

The tightly woven collagen fibres of the inner collagenous layer and the dense elastic layer by their appearance can be surmised to act as a flexible yet stable platform in 2 dimensions to support the retina, much as the plastic film base acts as a support for the emulsion in photographic film. Another function that can be hypothesised is that these layers act as a mechnical barrier that keeps the choriocapillaries away from the retina. Fig. 16 represents an artist's concept of the composite findings of scanning and transmission electron microscopy.

Dale Deg assisted in devising techniques to expose the layers in Bruch's membrane.

This work was supported in part by grant NIH EY 02434 and VA grant MRIS 3192

\section{References}

1 Bruch KLW. Untersuchungen zur Kenntnis des Kornigen Pigments der Wirbeltier. Zurich: Diss, 1844.
2 Wolfrum M. Beitrage zur Anatomie und Histologie der Aderhaut beim Menschen und bei hoheren Wirbeltieren. Albrecht von Graefes Arch Klin Ophthalmol 1908; 67: 307-59.

3 van den Hoof A. Het elektronen-microscopische beeld van het membraan van Bruch. Ned Tijdschr Geneeskd 1954;98: 2869-70.

4 Verin P. Gendre P, Le Rebeller M. Bruch's membrane and its connection with the choriocapillary. Arch Ophtalmol (Paris) 1969; 29: 123-34.

5 Sumita R. The fine structure of Bruch's membrane of the human choroid as revealed by electron microscopy. J Electron Microsc (Tokyo) 1961; 10: 111-8.

6 Nakaizumi Y. The ultrastructure of Bruch's membrane in human. monkey, rabbit, guinea pig, and rat eyes. Arch Ophthalmol 1964; 72: $380-7$.

7 Nakaizumi Y, Hogan MJ, Feeney L. The ultrastructure of Bruch's membrane. III. The macular area of the human eye. Arch Ophthalmol 1964; 72: 395-400.

8 Hogan MJ. Electron microscopy of Bruch's membrane. Trans Am Acad Ophthalmol Otolaryngol 1965; 69: 683-90.

9 Spitznas M. The fine structure of the chorioretinal border tissues of the adult human eye. Adv Ophthalmol 1974; 28: 78-174.

10 Wickham MG, Worthen DM. Correlation of scanning and transmission electron microscopy on the same tissue sample. Stain Technol 1973; 48: 63-8.

11 Sakuwagara M, Kuwabara T. The pigment epithelium of the monkey. Topographic study by scanning and transmission electron microscopy. Arch Ophthalmol 1976; 94: 285-92.

12 Angelucci A. Histologische Untersuchungen uber das retinale Pigment Epithel der Wirbeltiere. Arch Physiol Lpz 1878: 358-86.

13 Verhoeff FH. A hitherto undescribed membrane of the eye and its significance. $R$ Lond Ophthalmic Hosp Rep 1903; 15: 309-19.

14 Shiose Y. Electron microscopic studies on blood-retinal and blood aqueous barriers. Jpn J Ophthalmol 1970; 14: 73-87.

15 Peyman GA. Spitznas M, Straatsma BR. Peroxidase diffusion in the normal and photocoagulated retina. Invest Ophthalmol Visual Sci 1971; 10: 181-7.

16 Peyman GA. Spitznas M, Straatsma BR. Chorioretinal diffusion of peroxidase before and after photocoagulation. Invest Ophthalmol Visual Sci 1971; 10: 487-95.

17 Farquhar MG, Palade GE. Junctional complexes in various epithelia. J Cell Biol 1963; 17: 375-412. 